\title{
Maximum power's transfer and conformal transformations in the complex plane
}

\author{
F. De la Bodega ${ }^{1}$, M.T. Bedialauneta ${ }^{2}$, Z. Aginako $^{3}$ \\ Department of Electrical Engineering \\ Escuela Universitaria de Ingeniería Técnica Industrial de Bilbao, Pz. La Casilla nº 3, 48012 Bilbao \\ e-mail: faustino.delabodega@ehu.es ${ }^{1}$, miren.bedialauneta@ehu.es², zaloa.aginako@ehu.es³.
}

\begin{abstract}
.
The aim of this communication is to give in a simple and concise, but at the same time clear way, the keys of the maximum power's transfer or available power.

Taking into account the character of this paper, firstly we will develop the how and why is interesting the extraction of this power and which will be its maximum value.

Secondly, we will analyse the value of the lost power in any load, its different analytical expressions and the ways of its possible changes through the different parameters modification or variation

Finally, we will obtain the necessary conditions to achieve the maximum power transfer, distinguishing between those which has not got any restriction and those which are conditioned by any design-characteristic. Additionally it will be indicated how the practice can be carried out.
\end{abstract}

To sum up, and as a reminder, we can extract some conclusions related to the made study and which will be of interest

With the given references, we can considerer the study finished.

\section{Key words}

Transfer, power, circuits, analysis, theorems

\section{Power Transfer to a load in a circuit}

We wanted to know the average, active, or effective power "P" transferred to any load, $\underline{Z}=R+j X=Z \angle \varphi$, connected to a linear and active circuit (L.A.C.). The possible solutions are shown here:

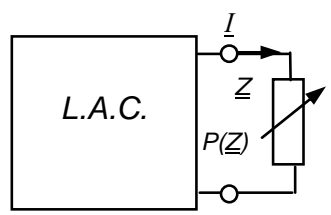

Fig. 1.a. L.A.C.

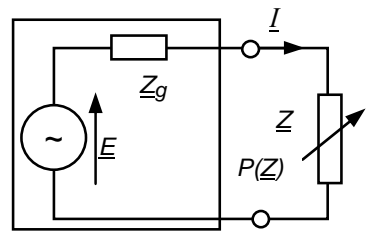

Fig. 1.b. Thevenin's equivalent
When $\mathrm{Z}$ is constant it is very easy: the value of the current, $I$, is obtained by any analysis method being the solution immediate:

$$
\begin{aligned}
& \underline{Z}=R+j X \Rightarrow P=R \cdot I^{2} \\
& \underline{Z}=Z \angle \varphi \Rightarrow P=Z \cdot \cos \varphi \cdot I^{2}
\end{aligned}
$$

If $\mathrm{Z}$ is variable the previous procedure is unfeasible, or at least, difficult to solve, because there are infinite solutions, being the power, $\mathrm{P}$, function of the load $\mathrm{Z}$ : "P(Z)". In this case, to apply Thevenin's theorem to the rest of the circuit seen from Z's terminals, in this case it is an advantage to apply. The obtained equivalent (Thevenin's equivalent) connected to de load constitute a series circuit, Fig. 1.b, which is easier to study.

This second case is the one that is going to be considered.

\section{Expressions of power transferred to $\underline{Z}$}

Power transferred to any impedance:

$\underline{Z}=R+j X=Z \angle \varphi$ :

If $\underline{Z}$ is given in binomial form: $\underline{Z}=R+j X$, power: $P=R \cdot I^{2}$ being the current:

$$
I=\frac{E}{|\underline{Z g}+\underline{Z}|}=\frac{E}{Z t} \quad \text { and } \quad I^{2}=\frac{E^{2}}{Z t^{2}}
$$

If we represent on the impedances' plane $\underline{Z}, P(\underline{Z}), \underline{Z g}, \underline{Z}$ and $\underline{Z} t$ Fig. 2.

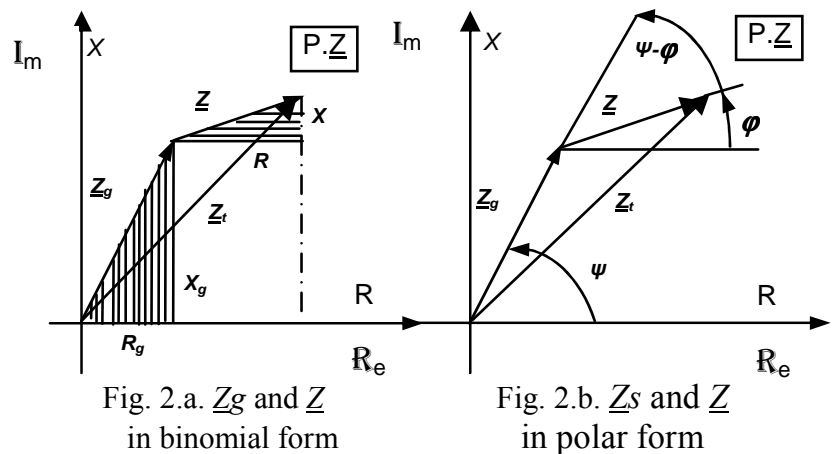


And apply Pythagoras' theorem to Fig. 1.a, $Z_{t}^{2}=\left(R_{g}+R\right)^{2}+\left(X_{g}+X\right)^{2}$ will be obtained, and consequently:

$$
P=E^{2} \cdot \frac{R}{\left(R_{g}+R\right)^{2}+\left(X_{g}+X\right)^{2}}
$$

If $\underline{Z}$ is given in polar form: $\underline{Z}=Z \angle \varphi$

$P=Z \cdot \cos \varphi \cdot \frac{E^{2}}{Z_{t}^{2}}$

Applying cosine's theorem to the figure, Fig. 2.b $Z_{t}^{2}=Z_{g}^{2}+Z^{2}+2 \cdot Z_{g} \cdot Z \cdot \cos (\psi-\varphi)$

therefore:

$$
P=E^{2} \cdot \frac{Z \cdot \cos \varphi}{Z_{g}^{2}+Z^{2}+2 \cdot Z_{g} \cdot Z \cdot \cos (\psi-\varphi)}
$$

This is the expression of power, transferred to $Z$.

\section{Extreme values of transferred power.}

As it has been seen, how the value of the power $P$ transferred to any impedance, $\underline{Z}$, is function of two variables. $\underline{Z}=R+j X$, of $R$ and $X$ or $\underline{Z}=Z \angle \varphi$, of $Z$ and of $\varphi$.

And it will be also when the power to be transferred is maximum $\hat{P}$.

Two Maximum types will be considered, "conditioned" and "Free Maximum".

\section{A. Conditioned Maximum}

The maximum will be called conditioned or relative when a constraint is imposed to $\underline{Z}$. Such as, that only one parameter $R$ or $X$ can vary, or, if the two of them vary, it must be in a dependent way as:

$X=k \cdot R \quad(k=c t e)$.

In the complex plane $\underline{Z}(\mathrm{P} . \underline{Z})$ they are represented by their respective loci of $\underline{Z}$ ( $\underline{Z}$ G.L) Fig. 3 .

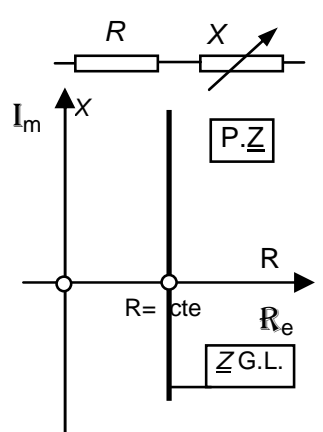

Fig. 3.a. $R=$ cte

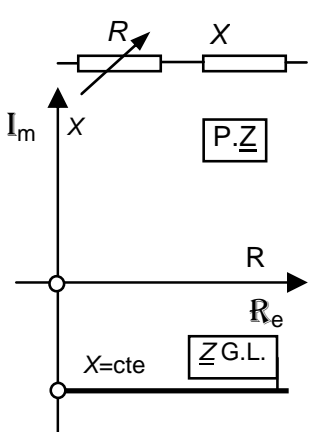

Fig. 3.b. $X=$ cte

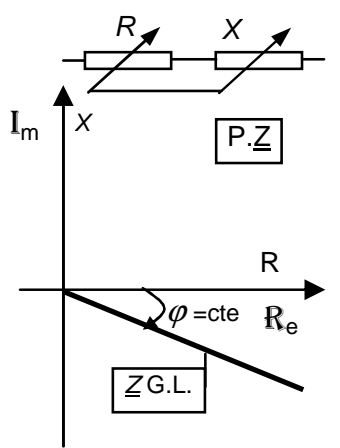

Fig. 3.c. $\varphi=$ cte.

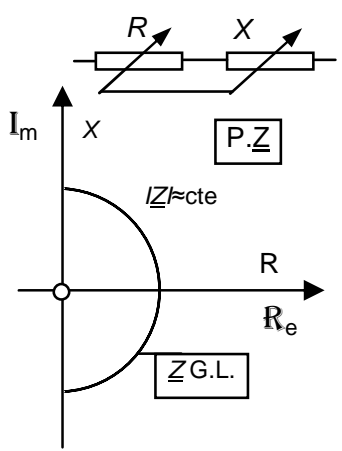

Fig. 3.d. $Z=$ cte
Thus $\underline{Z}$ G.L. varies:

$X \quad$ it is a parallel line to the imaginary axis. Fig. 3.a.

$R \quad$ it is a half-line parallel to the real axis. Fig. 3.b.

$Z \quad$ it is a half-line that departs from the origin Fig. 3.c.

$\varphi \quad$ it is a semicircumference with the center in the origin. Fig. 3.d.

\section{B. Free Maximum}

The maximum will be free, absolute or "maximum maximorum" when no constraint is imposed to $\underline{Z}$, that is, when $R$ and $X$ can vary in a free and independent way, it is the same to say that $Z$ and $\varphi$ are which vary. The free maximum is represented by the domain of $\underline{Z}$ (D. $\underline{Z}$ ) on the Z.P. It is made up of the right halfplane whose border is the imaginary axis Im, and, which is divided by the real axis $\mathbf{R e}$ in the I and IV quadrants, where any impedance can be represented. Fig. 4.

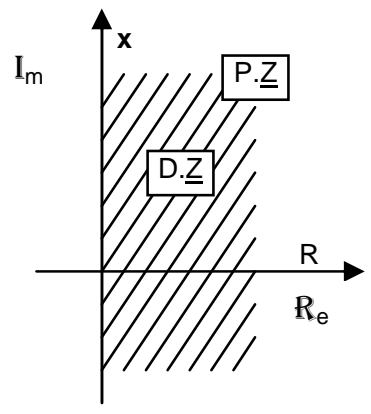

Fig. 4. D. $\underline{Z}$

\section{Conditioned Maximum}

The study begins obtaining the values of $\hat{P}$ and the $\underline{Z}$ which leads to achieve the maximum power $(\hat{P})$ considering the different conditions imposed to $\underline{Z}$. Later these values will be obtained when $\underline{Z}$ is free.

A. The resistance " $R$ " remains constant and the reactance " $X$ " varies.

Considering the circuit represented in Fig. 5, the G.L. $\underline{Z}$ is a line, parallel to the imaginary axis Fig. 5.b, it will be 
also the locus of total $\underline{Z}$ (G. L $Z_{\mathrm{t}}$ ), being $\underline{Z}_{t}=\underline{Z}_{g}+\underline{Z}$, Fig. 5.c.

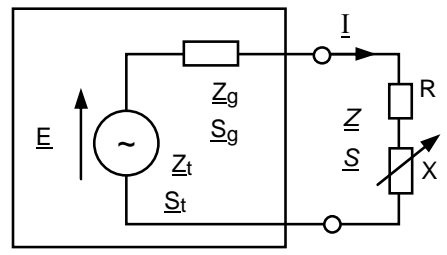

Fig. 5.a. $\underline{Z}=R+j X \quad \mid-\infty \leq X \leq \infty$

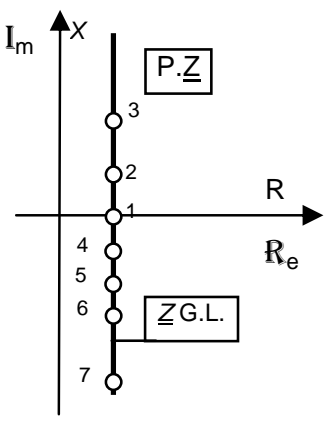

Fig. 5.b. ZGG.L.

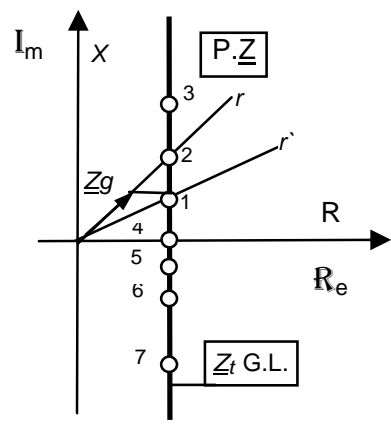

Fig. 5.c. $\underline{Z} t$ G.L.
As a result, the locus of its conjugate admittance $\underline{\mathrm{Y}}_{t}^{*}$, $\left(\underline{Y}_{t}^{*}\right.$ G.L.) is a circumference with center in the real axis, that contains the origin "O" and, whose diameter is: $d=1 /\left(R_{g}+R\right)$.

To each value of $\underline{Z}$ corresponds another of $\underline{Z}_{t}$, and other one of $\underline{\mathrm{Y}}_{t}^{*}$. In the same way the locus of the apparent total power $\underline{S}_{t}$ (L.G. $\underline{S}_{t}$ ) is a circumference homothetic to the previous one ( $\underline{\mathrm{Y}}_{t}^{*}$ G.L.), with center of similitude "O" and homothetic ratio $U^{2}$, being $\underline{S}_{t}=U^{2} \cdot \underline{\mathrm{Y}}_{t}^{*}$, just as it is shown in Fig.6

On the other hand the locus of the power lost in $\underline{Z}_{g}, \underline{S g}$ ( $\underline{S g}$ G.L.) must be the segment of line that contains $\underline{Z}_{g}$ because its apparent power is: $\underline{S}_{g}=\underline{Z}_{g} \cdot I^{2}$.

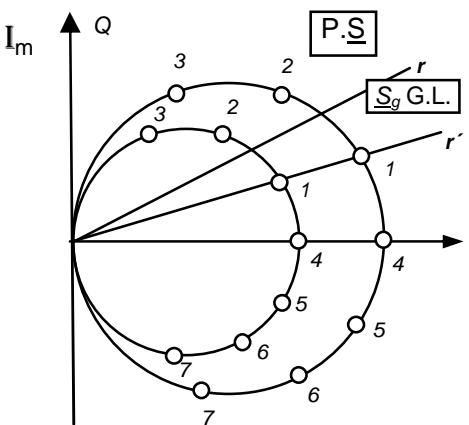

Fig.6 $\underline{Z}_{t}$ G.L. $\underline{\mathrm{Y}}_{t}^{*}$ and $\underline{S}_{t}$ if $\underline{Z}=R+j X \quad \mid-\infty \leq X \leq \infty$.
Consequently, $\underline{S}$, the power lost in $\underline{Z}$, will be the difference between the total, $\underline{S}_{t}$, and the one lost in $\underline{Z}_{g}$, $\underline{S}_{g}$

The previous, is reflected graphically in the figures Fig. 7.a and Fig. 7.b

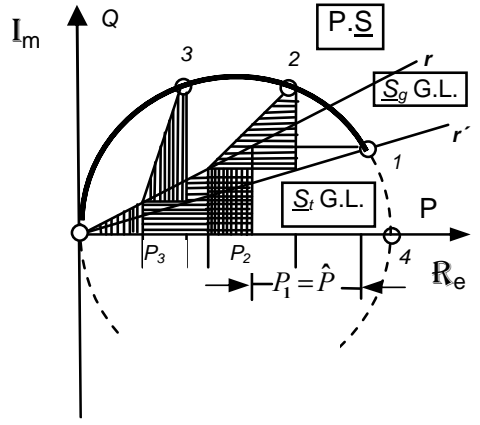

Fig. 7.a. $X$ inductive

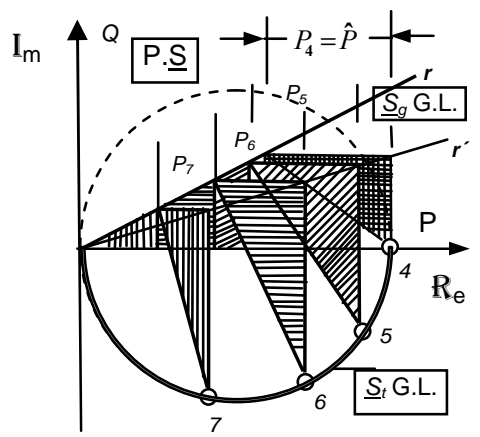

Fig. 7.b. $X$ capacitive

From those figures can be deduced the next:

- If $X$ and $X_{g}$ are both of the same nature, $\hat{P}$ will be obtained when: $X=0$, so

$$
\underline{Z}=R+j 0
$$

And from the equation (1):

$$
P=E^{2} \cdot \frac{R}{\left(R_{g}+R\right)^{2}+X_{g}^{2}}
$$

- If $X$ can take any value, or $X$ and $X_{g}$ are not both of the same nature, then :

$$
\begin{aligned}
& X=-X_{g}, \text { so: } \\
& \underline{Z}=R-j X_{g}
\end{aligned}
$$

and

$$
\hat{P}=R \cdot \frac{E^{2}}{\left(R_{g}+R\right)^{2}}
$$

It must be mentioned that the effort made would only be worth because it provides the method to follow in the studied cases below.

The answer is indeed clear: if $R$ resistance is constant, the power transferred to it, is maximum only when current is maximum too, and this happens when the only term that can vary " $\mathrm{Xg}+\mathrm{X}$ " is minimum. This is what happens in the studied previous cases. 


\section{B. The argument " $\varphi$ " remains constant, and the module " $Z$ " varies.}

In this case the G.L. $\underline{Z}$ is a half-line that contains the origin, and makes with the real axis a " $\varphi$ " angle Fig. 8.b, although G.L. $\underline{Z} t$ is the previous one, to which it has applied a $\mathrm{Zg}$ translation, being consequently a half-line parallel to the G.L.ZZt Fig. 8.c.

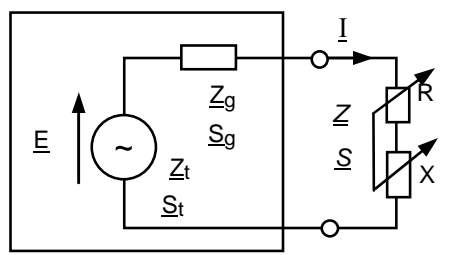

Fig. 8.a. $\underline{Z} \mid X / R=$ cte $\quad \wedge 0 \leq Z \leq \infty$

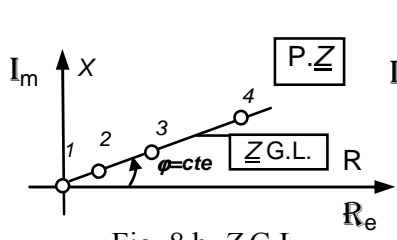

Fig. 8.b. $\underline{Z}$ G.L.

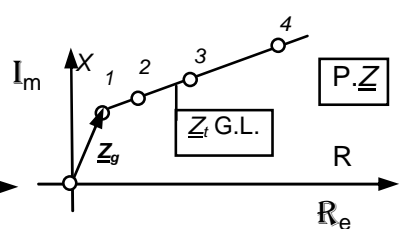

Fig. 8.c. $\underline{Z} t$ G.L.
Following the dynamic of the previous case, the $\underline{\mathrm{Y}}_{t}^{*}$ G.L. will be a circumference that contains the origin "O", whose center is in a line that contains the origin and that is perpendicular to $\underline{Z} \mathrm{t}$ G.L.

The diameter of the circumference is: $d=1 / \overline{O A}$ or $d=1 / Z g \sin (\psi-\varphi)$ Fig.9.

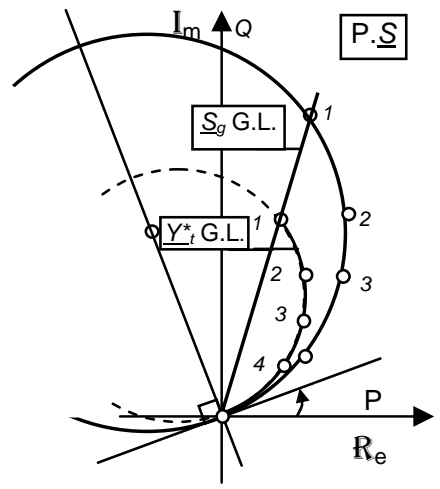

Fig. 9.

In the same way the $S t$ G. L. is homothetical to the previous one with center of similitude "O" and a ratio of similitude $U^{2}$. Finally the $\underline{S g}$ G. L. will be the segment that contains $\underline{Z g}$.

Fig. 10 shows the different powers. As it is known $P$ must be maximum, due to this imposed condition, the other powers $Q$ and $S$ will be maximum too, being $S=P / \cos \varphi$ and $Q=P \cdot \operatorname{tag} \varphi$.
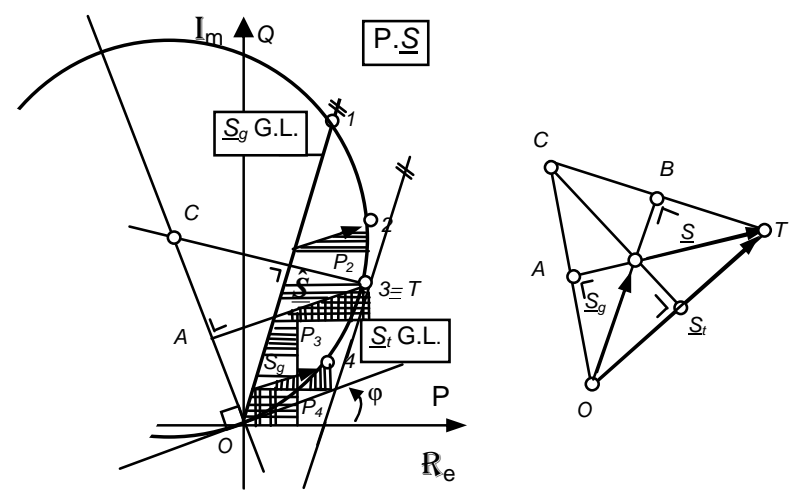

Fig. 10

We are going to study when $\underline{S}$ is maximum.

Watching at Fig. 10 it can see that $\underline{S}$ is maximum in point $\mathrm{T}$, tangent to the circumference, with a line with the same slope than $\underline{\boldsymbol{S}}_{g}$.

In these conditions $O C T$ triangle is isosceles, because $\overline{O C}$ and $\overline{C T}$ are both radius of the circumference and, as a result, equals.

On the other hand, $\overline{O B}$ and $\overline{C T}$ are perpendicular segments, because they are a radius and a tangent line. Thus $\overline{O B}$ match with the height traced from $O$

In the same way $\overline{O C}$ and $\overline{T A}$ are both perpendicular lines. Because that is the condition to obtain the $\underline{\mathrm{Y}}_{t}^{*}$ G.L. Thus $\overline{T A}$ is the height traced from $T$.

From previous conditions it is easy to deduce that the requirement to obtain a maximum $\underline{S}$ is: $\hat{S} \cong S g$ which implies that $Z \cdot I^{2}=Z_{g} \cdot I^{2}$

$$
Z=Z_{g} \quad \text { or } \quad Z=\sqrt{R_{g}^{2}+X_{g}^{2}}
$$

And the maximum power according to (2) will be:

$$
\begin{aligned}
& \hat{P}=Z_{g} \cdot \cos \varphi \cdot=\frac{E^{2}}{2 \cdot Z_{g}^{2}+2 \cdot Z_{g}^{2} \cos (\psi-\varphi)}= \\
& E^{2} \frac{\cos \varphi}{2 \cdot Z_{g}[1+\cos (\psi-\varphi)]}
\end{aligned}
$$

So that:

$$
\begin{aligned}
& \cos ^{2} \frac{\psi-\varphi}{2}=\frac{1+\cos (\psi-\varphi)}{2} ; \\
& \hat{P}=E^{2} \frac{\cos \varphi}{4 \cdot Z_{g} \cos ^{2} \frac{\psi-\varphi}{2}}
\end{aligned}
$$


As a conclusion, the maximum power and the impedance that gets will be:

$\underline{Z}=Z_{g} \angle \varphi$ o $\underline{Z}=\sqrt{R_{g}^{2}+X_{g}^{2}} \angle \varphi$

And

$\hat{P}=E^{2} \frac{\cos \varphi}{4 \cdot Z_{g} \cos ^{2} \frac{\psi-\varphi}{2}}=E^{2} \frac{\cos \varphi}{2 \cdot Z_{g}[1+\cos (\psi-\varphi)]}$

\section{The impedance is a variable resistance:}

It is a particular case of the previous one, whose $\varphi=0, R=Z \angle 0^{\circ}$, just as it is shown in Fig. 11. And as a result:

$R=Z_{g}$ o $R=\sqrt{R_{g}^{2}+X_{g}^{2}}$

And as $\varphi=0 ; \cos 0=1$, then:

$$
\hat{P}=E^{2} \frac{1}{4 \cdot Z_{g} \cos ^{2} \frac{\psi}{2}}
$$

or

$$
\hat{P}=E^{2} \frac{1}{2 \cdot Z_{g}[1+\cos \Psi]}
$$

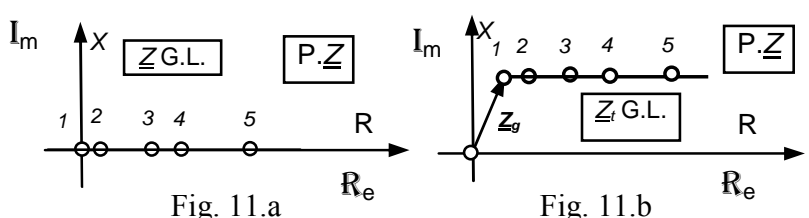

Fig. 11.a

Fig. 11.b

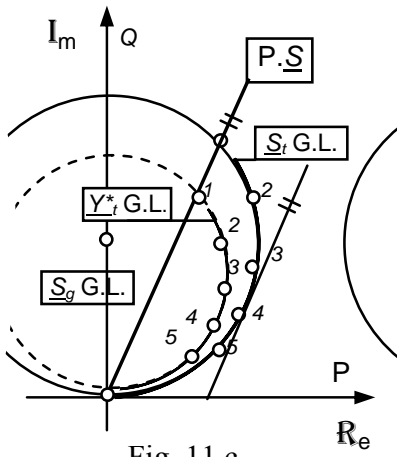

Fig. 11.c

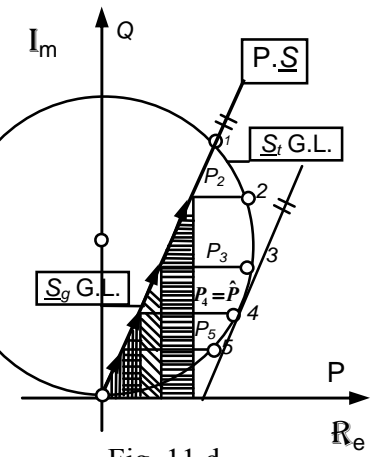

Fig. 11.d
$D$. The reactance " $X$ " remains constant and the resistance " $R$ " varies:

It can be reduced to the previous case, see the circuit of Fig. 12.a. The Z.G.L. is a line parallel to the real axis Fig. 12.b and also it is the $\mathrm{Zt} \mathrm{G.L.} \mathrm{Fig.} \mathrm{12.c}$

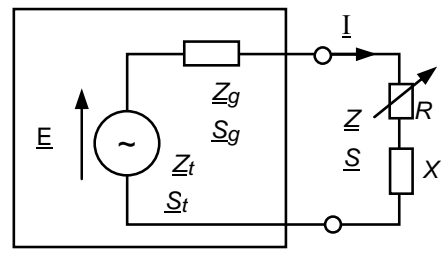

Fig. 12.a

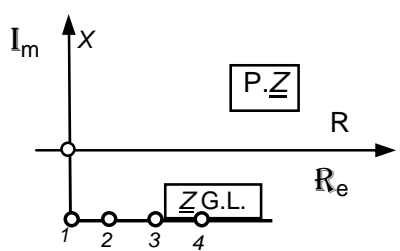

Fig. 12.b G.L. $\underline{Z}=R+j X \quad \mid \mathbf{0} \leq R \leq \infty$

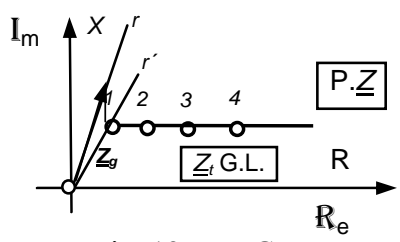

Fig. 12.c. $\underline{\text { t }}$ G.L.

If we consider $\underline{Z}_{g}^{\prime}=\underline{Z}_{g}+X=R_{g}+j\left(X X_{g}+X\right)$ we have a circuit with $\underline{Z}_{g}^{\prime}$ and $R$ variable, so:

$\underline{Z}=Z_{g}^{\prime}+j X$

Or

$$
\begin{aligned}
& R=Z_{g}^{\prime}=\sqrt{R_{g}^{2}+\left(X_{g}+X\right)^{2}} \text { and, as a result: } \\
& \underline{Z}=\sqrt{R_{g}^{2}+\left(X_{g}+X\right)^{2}}+j X
\end{aligned}
$$

And $\hat{P}=E^{2} \cdot \frac{1}{4 \sqrt{R_{g}^{2}+\left(X_{g}+X\right)^{2}} \cdot \cos ^{2} \frac{\Psi^{\prime}}{2}}$

$$
\hat{P}=E^{2} \cdot \frac{1}{2 \sqrt{R_{g}^{2}+\left(X_{g}+X\right)^{2}} \cdot\left(1+\cos \Psi^{\prime}\right)}
$$

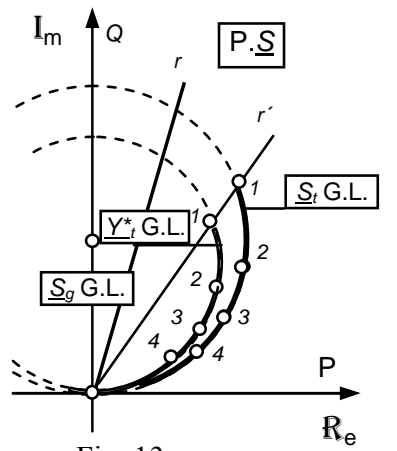

Fig. 13.a

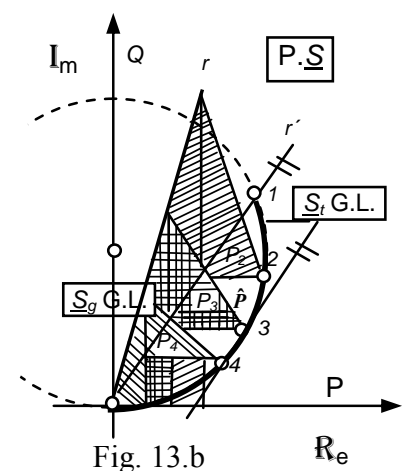




\section{Free maximum}

Before going further, we will summarize the conditioned maximums already seen, because they are the base to obtain the free maximum. The condition that $\underline{Z}$ must fulfil, if it could vary in the ways seen before, is:

$$
\begin{aligned}
& \text { In (5): } \quad \underline{Z}=R+j X \mid R=\text { cte } \wedge \mathbf{X} \in \mathbf{R}_{\mathrm{e}} ; \\
& \hat{P} \Leftrightarrow X=-X_{g} \wedge \underline{Z}=R-j X_{g} \\
& \text { In } \quad(13): \quad \underline{Z}=R+j X_{g} \mid R \in \boldsymbol{R}_{e}^{+} \wedge X=\text { cte } ; \\
& \hat{P} \Leftrightarrow R=\sqrt{R_{g}^{2}+\left(X_{g}+X\right)^{2}} \\
& \text { In } \quad(7): \quad \underline{Z}=Z \angle \varphi \quad \mid Z \in \boldsymbol{R}_{e}^{+} \wedge \varphi=c t e ; \\
& \hat{P} \Leftrightarrow Z=Z_{g} ; \\
& Z=\sqrt{R_{g}^{2}+X_{g}^{2}} \wedge \underline{Z}=Z_{g} \angle \varphi \wedge Z=\sqrt{R_{g}^{2}+X_{g}^{2}} \angle \varphi
\end{aligned}
$$

Well, when a free $\underline{Z}$ is required, that is $\underline{Z}=R+j X$ $R \in \boldsymbol{R}_{\boldsymbol{e}}^{+} \wedge X \in \boldsymbol{R}_{\boldsymbol{e}}$, without any constraint,

Then at the same time it should fulfil 5 and 13 conditions, or 5 and 7. Let see it:

If (5): $X=-X_{g}$

$$
\begin{aligned}
& \text { If } \quad(13) \quad R=\sqrt{R_{g}^{2}+\left(X+X_{g}\right)^{2}} \\
& R=\sqrt{R_{g}^{2}+\left(-X_{g}+X_{g}\right)^{2}} \quad \text { or } \quad R=R_{g} \text { therefore } \\
& \underline{Z}=R_{g}-j X_{g} \text { o } \underline{Z}=\underline{Z}_{g}^{*}
\end{aligned}
$$

Arriving to the same conclusions with 5 and 7 conditions.

(5) $X=-X_{g}$

(7) $Z=Z_{g}=\sqrt{R_{g}^{2}+X_{g}^{2}} \quad$ which entails $\varphi=-\Psi$

That is, $\underline{Z}=Z_{g} \angle-\Psi$ o $\underline{Z}=\underline{Z}^{*}{ }_{g}$

And the maximum power transferred will be:

from (1)

$\hat{P}=R_{g} \cdot \frac{E^{2}}{\left(R_{g}+R_{g}\right)^{2}+\left(X_{g}-X\right)^{2}}=\frac{E^{2}}{4 R_{g}}$

from (2)

$$
\begin{aligned}
& \hat{P}=Z_{g} \cos \Psi \cdot \frac{E^{2}}{Z_{g}{ }^{2}+Z_{g}{ }^{2}+2 Z_{g} Z_{g} \cos 2 \Psi}= \\
& \frac{E^{2} \cos \Psi}{2 Z_{g}[1+\cos 2 \Psi]}
\end{aligned}
$$

Or better, from (9):

$$
\frac{E^{2} \cos \Psi}{4 Z_{g}\left[1+\cos ^{2} \frac{2 \Psi}{2}\right]}=\frac{E^{2}}{4 Z_{g} \cos \Psi}
$$

\section{Conclusions}

As it has been seen, the calculation of the maximum power transfer is itself a classical problem, studied by the technique of function optimization. However, the analysis has been carried out in a completely different and original way.

The study has been carried out, basing on techniques of "Conformal transformations on the complex plane" such as inversions, similitudes, etc, by observing the loci of powers at stake.

The answer is obtained graphically in an easy way, and in order to get numerical values only the elementary Euclidean geometry is required.

In addition, the proposed method has an added value against the classic. Not only it shows the variable element value that gets the maximum power or available power itself. It also provides the behaviour of all of the powers (active power, reactive power and apparent power) in the considered element, and what, and how they vary depending on the variable element.

Lastly and from a didactic point of view, it is important to highlight that the needed mathematical apparatus is extremely simple.

\section{References}

[1] L. Serrano Iribarregaray, Energía Solar fotovoltaica, Mundo. Ed. Marcombo, Barcelona (1983), pp. 137-147

[2] Leon O. Chua, Carles A. Desoer, Ernest S. Kuh. Linear and no linear circuits, Ed. Mac Graw Hill, New York (1987), pp. $532-54$

[3] Enrique Ras Oliva, Teoría de Circuitos, Ed. Marcomobo, Barcelona (1999), pp. 205-215

[4] Luis Gaspar Vega Argüelles, Transformaciones Conformes en el plano Complejo y su aplicación al campo de la Electrotecnia (Lugares geométricos) Doctoral thesis, University of the Basque Country (UPV/EHU) Bilbao 1982, pp. 45-80 